\title{
Searching Visual Media Service Providers Using ASN.1-Based Ontology Reasoning
}

\author{
Youngkun Min, Bogju Lee, and Yunmook Nah \\ Department of Computer Engineering, Dankook University \\ Hannam Dong, Youngsan Ku, Seoul, Korea \\ \{minyk, blee, ymnah\}@dankook.ac.kr
}

\begin{abstract}
Information retrieval is one of the most challenging areas in which the ontology technology is effectively used. Among them, image retrieval using the image metadata and ontology is the one that can substitute the keywordbased image retrieval. In the paper, the retrieval of visual media such as the art image and photo picture is handled. It is assumed that there are more than one service providers of the visual media, and also there is one central service broker that mediates the user's query. Given the user's query the first step that must be done in the service broker is to get the list of candidate service providers that fit the query. This is done by defining various ontologies such as the service ontology and matching the query against the ontology and providers. A novel matching method based on the ASN.1 is proposed in the paper. The experiment shows that the method is more effective than the existing tree-based or interval-based methods.
\end{abstract}

\section{Introduction}

Image retrieval from the web is an important research issue. The technology has been is improved from the exact query matching and content-based retrieval using general features such as colors, textures, shapes of the images, to the semantic content-based retrieval using concepts, semantics, categories and spatial relationships of images. To accomplish the semantic content-based retrieval the ontology is effectively used.

There are several researches on the image retrieval using the ontology in distributed environment. They include the Finnish Museums on the Semantic Web [1] at Finland HIIT (Helsinki Institute for Information Technology), Semantic Annotation of Image Collections [2] at University Amsterdam Computer Science, and SIMILE Project [3] from W3C, HP, MIT Libraries, and MIT CSAIL. Research goal at HIIT is a web portal site for heterogeneous databases that have different table schema and retrieval method. For the goal they need unification of data. So they build syntactic interoperation using XML-schema and use RDFS-RDF for semantic relation. The site supports view-based image retrieval for more useful user-interface to end user, and recommended services for intelligent service. In University Amsterdam Computer Science, they perform the research on describing images using the existing ontology such as WordNet. They are focusing on building ontology for images and describing metadata. The goal of SIMILE project is to develop a system to integrate distributed 
images that a person or community has. It provides the service search with ontology and schema metadata for user.

In this paper, it is assumed that there are multiple image providers and single central broker in a distributed environment. The broker accepts and answers the user's query. The provider's services are classified into the service ontology. Each provider has its own provider-specific service ontology and the broker has the union of all the providers' service ontology. Given a user's query, the broker needs to find the most appropriate providers that answer the query quickly and effectively.

The existing query matchmaking methods include the well-known CMU's matchmaking method [4] and the interval-based matchmaking which is proposed by the Swiss Federal Institute of Technology [5]. In this paper we suggest a new matchmaking method based on the ASN.1 scheme. The experiment shows that our method is more effective than the tree-based (CMU's matchmaking) and the interval-based method.

Section 2 describes the two existing matchmaking methods. Section 3 introduces our service ontology and the ASN.1-based matchmaking method. Section 4 shows the experimental result and finally Section 5 gives the conclusion.

\section{Existing Matchmaking Methods}

As in the figure 1, there are four matchmaking patterns between query service $\mathrm{Q}$ and library service $\mathrm{S}$. "Exact" pattern means the library service $\mathrm{S}$ is matched to query service Q. "Plug in" means library service $S$ is plugged in the query service Q. "Subsume" means the library service $\mathrm{S}$ is subsumed within the query service $\mathrm{Q}$. "Failed" means there is no relationship between the library service S and the query service Q. Order of matchmaking estimation pattern is firstly "Exact", secondly "Plug In", thirdly "Subsume", and finally "Failed", so the "Failed" has the lowest estimation pattern [6].

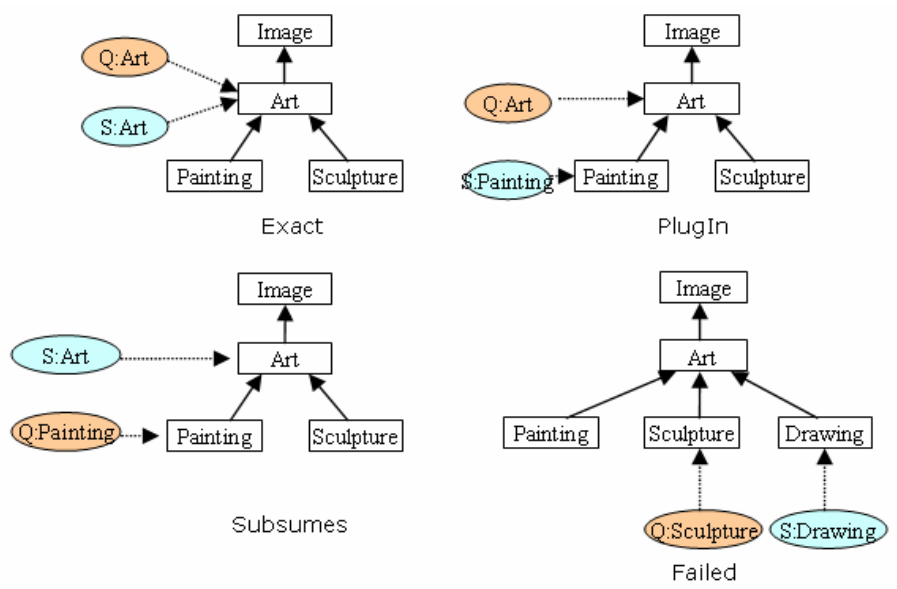

Fig. 1. Matchmaking estimation patterns 
Interval-based matchmaking method is suggested by Swiss Federal Institute of Technology, any class in multi-inheritance layer can be symbolized into interval and tree. The interval is included in other intervals but not overlapped. They use two standards for setting up interval values. One is parent-child relationship and the other is child-child relationship. In parent-child relationship, interval is determined at between 0 and 1. Child node is assigned unique key from parent node and interval with key-dependence function. Namely, parent-node is assigned global interval, childnode interval that is connected it is there into parent-node's global interval [5].

In the visual media service ontology, for example, Visual Media is represented by $<0,1\rangle$. Then its children Video and Image are represented by $<0,0.5\rangle$ and $\langle 0.5,1\rangle$. Image's children Art, Medical, and Photo are represented by $<0.5,0.6\rangle,<0.6,0.7\rangle$, $<0.7,0.8>$. So Art domain is sub-domain of the Visual Media domain since $<0.5,0.6\rangle$ (Art) is included by $<0,1>$ (Visual Media). Also, Art domain has no inclusion relationship with Video domain since $<0.5,0.6>$ (Art) has no overlap with $<0,0.5>$ (Video).

This method, however, has problems in that there cannot be more than ten children since it uses the floating point number. Also the number of digits increases as the tree depth increases.

\section{Visual Media Service Ontology and ASN.1-Based Matchmaking}

Visual media service ontology is defined and used as a part of HERMES Visual Media Retrieval System [7]. As described in Section 1. The broker accepts and answers the user's query. Each provider has its own provider-specific service ontology and the broker has the union of all the providers' service ontology. Given a user's query, the broker needs to find the most appropriate providers that answer the query quickly and effectively.

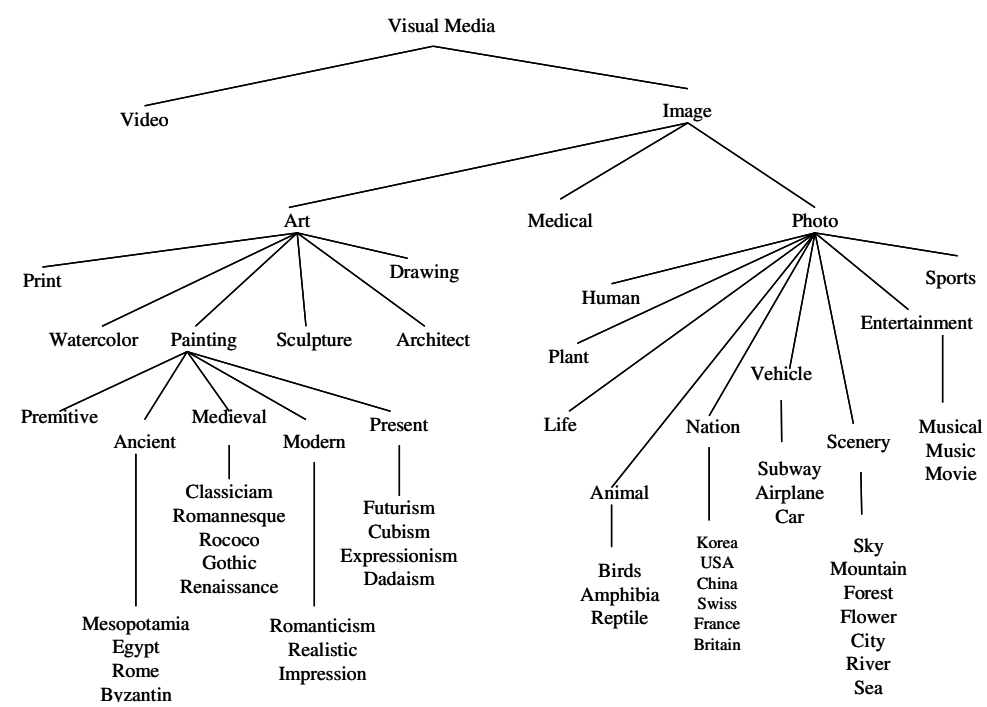

Fig. 2. The visual media service ontology 
Visual media service ontology provides a whole classification domain for the provider's services. Figure 2 shows the service ontology which is used in the paper. Note that this is the union of all the providers' service ontology.

Now we explain the ASN.1-based matchmaking. ASN.1 standard [8], made by ISO, is the common abstract grammar to define data at distributed environment. All services have unique service ID's and we know easily the relationship between superconcept (parent) or sub-concept (child). This method has no limitation in terms of the number of children. Figure 3 shows the service ontology with ASN.1.

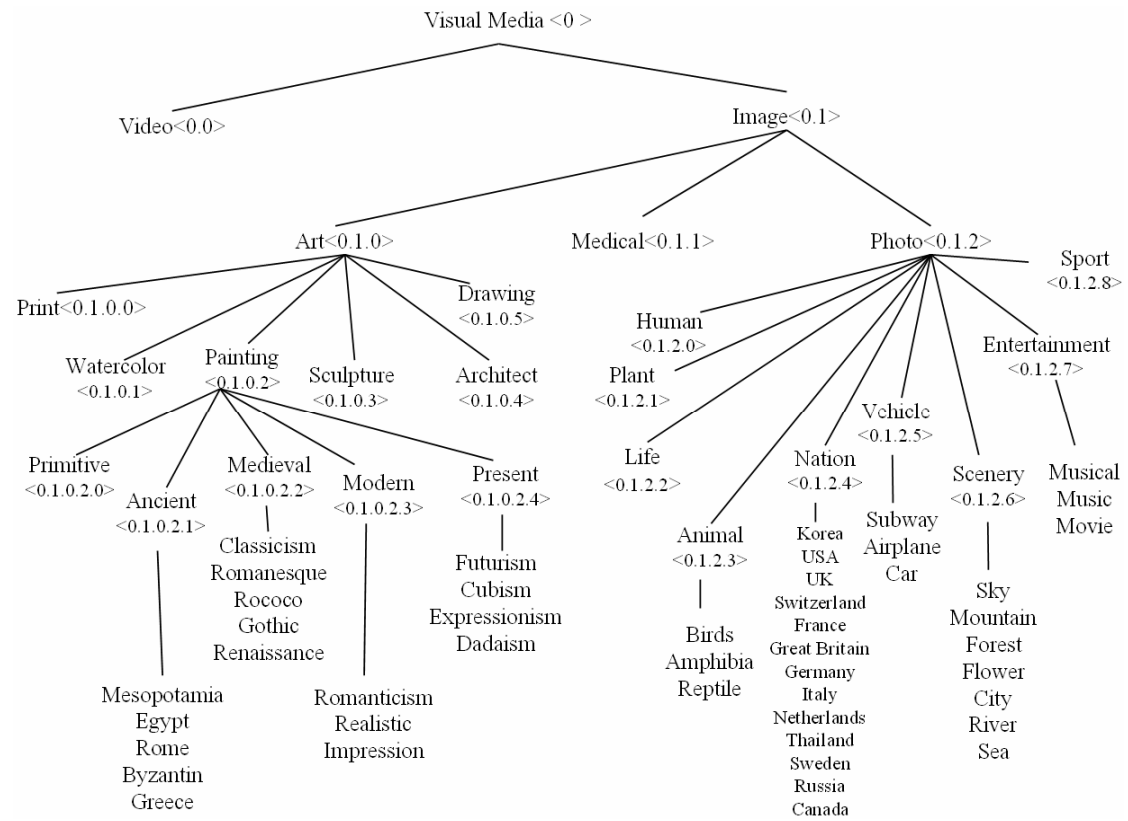

Fig. 3. The visual media service ontology with ASN.1

In this scheme, the root node Visual Media is represented by $<0>$. Its children Video and Image are represented by $<0.0>$ and $<0.1>$ respectively. Image's children Art, Medical, and Photo are represented by $\langle 0.1 .0\rangle,\langle 0.1 .1\rangle$, and $\langle 0.1 .2\rangle$ respectively. As for Print, Watercolor, Painting, Sculpture, Architecture, and Drawing which are Art's children, the numbers are given by adding numbers after $<0.1 .0\rangle$. So Print has $<0.1 .0 .0>$, Watercolor has $<0.1 .0 .1>$, Painting has $<0.1 .0 .2>$, Sculpture has $<0.1 .0 .3>$, Architecture has $<0.1 .0 .4>$, and Drawing has $<0.1 .0 .5>$. Suppose Q is the query domain ID, $\mathrm{P}$ is the provider service domain ID. Then the method determines the Exact, Plug In, and Subsume as follows.

Exact: $\mathrm{Q}=\mathrm{P}$

Plug In: $Q=\operatorname{prefix}(P)$

Subsume: $\operatorname{prefix}(\mathrm{Q})=\mathrm{P}$

Failed: None of above 
Plug In, for example, if $\mathrm{P}$ is $<0.1 .0 .2 .3>$ (Modern) and $\mathrm{Q}$ is $<0.1 .0 .2>$ (Painting), then since "0.1.0.2" (Painting) is a prefix of "0.1.0.2.3" (Modern), they have Plug In relationship. To effectively perform the matching, the provider services and their ID's are listed in a table. When the query is given it is matched against the table entries one by one. Exact, Plug In, Subsume, Failed are determined. The matched providers are listed in this order.

\section{Experimental Results}

The proposed ASN.1-based matchmaking is compared with the two existing methods, the tree traversal method and interval-based method. The experimental environments include Intel 3.0GHz CPU, 1GB RAM computer, Java 1.4.2_07, Tomcat 4.1, JENA 2.1, and My-SQL database.

Table 1 shows the number of providers after performing matchmaking with the three methods and four queries. As shown in the table, the tree traversal and our ASN.1 method give the same results. The interval-based method, however, has problem in returning extra providers which are incorrect.

Table 1. The number of providers after matchmaking

\begin{tabular}{|l|l|l|l|l|}
\hline & Modern & Scenery & Nation & Vehicle \\
\hline Tree traversal & 8 & 11 & 17 & 7 \\
\hline Interval-based & 8 & 11 & 13 & 10 \\
\hline ASN.1-based & 8 & 11 & 17 & 7 \\
\hline
\end{tabular}

Table 2 shows the execution times of the three methods. Our ASN.1 method has longer execution time than the interval-based method, but faster than the tree traversal method.

Table 2. The execution times (ms)

\begin{tabular}{|l|l|l|l|l|}
\hline & Modern & Scenery & Nation & Vehicle \\
\hline Tree traversal & 18.9 & 20.3 & 25.2 & 22.3 \\
\hline Interval-based & 6.5 & 4.87 & 7.14 & 3.87 \\
\hline ASN.1-based & 19.6 & 17.4 & 15.3 & 14.6 \\
\hline
\end{tabular}

As shown in the table 1 and 2, ASN.1-based method is slower than the intervalbased method, but more accurate than the interval-based method and faster than the tree traversal method, our method is more effective than the two other methods.

\section{Conclusions}

In the paper, we designed a service ontology which is used in retrieving visual media. To effectively find the service providers when the query is given, a novel ASN.1based matchmaking method is proposed. The proposed method is compared with 
existing methods in terms of accuracy and speed. The experimental result shows that the method is more effective that the existing methods. The ASN.1-based method can be used in any other domain.

\section{Acknowledgements}

This work was supported by grant No. R01-2003-000-10133-0 from the Basic Research Program of the Korea Science and Engineering Foundation.

\section{References}

1. Hyvonen, E., Junnila, M., Kettula, S., Saarela, S., Salminem, M., Syreeni, A., Valo, A., Viljanen, K.: Publishing Collections in the Finnish Museums on the Semantic Web Portal. In: Museums and Web Conference (MW 2004) (March 31 - April 1, 2004)

2. Hollink, L., Schreiber, G., Wielemaker, J., Wielinga, B.: Semantic Annotation of Image Collections. In: KCAP'03. Workshop on Knowledge Markup and Semantic Annotation, Florida (October 2003)

3. Semantic Interoperability of Metadata and Information in unLike Environments, http:// simile.mit.edu/

4. Paolucci, M., Kawamura, T., Payne, T.R., Sycara, K.P.: Semantic Matching of Web Services Capabilities. In: Proceedings of the First International Semantic Web Conference on the Semantic Web, pp. 333-347 (2002)

5. Constantinescu, I., Faltings, B.: Efficient Matchmaking and Directory Services. In: Proceedings of the IEEE/WIC International Conference on Web Intelligence (2003)

6. Choi, W., Yang, J., Choi, J., Cho, H., Cho, H., Kim, K.: Service Discovery Algorithm Using Ontoly Herarchy Relationship. The Korean Information System 30(1) (2003)

7. Kwon, E., Nah, Y.: Extended Query Processing using Image Metadata Mapping in Distributed and Heterogeneous Environments. In: SIGDB-KISS, pp. 250-257 (2005)

8. The ASN.1 Consortium, http://www.asn1.org/

9. The DARPA Agent Markup Language Homepage, http://www.daml.org

10. Protégé Ontology Editor and Knowledge Acquisition System, http://protege.stanford.edu/ overview/ 